\title{
Análise sobre o Termo Talento em Diferentes Áreas do Conhecimento
}

\author{
Bárbara Beatriz Freitag \\ Faculdade de Economia, Administração e Contabilidade da Universidade de São Paulo \\ (FEA/USP), Brasil \\ barbara-adm@usp.br \\ Andre Luiz Fischer \\ Faculdade de Economia, Administração e Contabilidade da Universidade de São Paulo \\ (FEA/USP), Brasil \\ afischer@usp.br
}

\section{RESUMO}

O mercado atual consiste em um ambiente global, complexo, altamente competitivo e volátil para as organizações, trazendo consigo desafios, também, globais de fluxo de talentos e escassez de competências essenciais. Daí a emergência da gestão de talentos como uma preocupação internacional e nacional de gestão de pessoas. Considerando-se o aspecto de multiplicidade e fluidez do mercado e de imprecisão do termo Talento em administração, surgiu o interesse de verificar a multidisciplinaridade desse termo. Então, emergiu o problema de pesquisa: Quais as características e as proposições de diferentes áreas do conhecimento acerca do termo Talento? A fim de colaborar com o debate sobre temas emergentes em gestão de pessoas, nesta pesquisa o objetivo consistiu em identificar características e proposições que outras áreas do conhecimento adotam para referir-se a Talento. Este estudo é uma revisão sistemática, realizada por meio de análise bibliométrica e análise de conteúdo. Foram analisados artigos acadêmicos selecionados na base Web of Science que continham o termo Talento em seu título. A leitura dos textos selecionados possibilitou a constatação de que, de acordo com essa amostra, os adjetivos mais utilizados para fazer menção e caracterizar os Talentos são os relacionados à diferenciação. A análise dos textos gerou a sugestão de dez proposições para futuras pesquisas. Este estudo foi desenvolvido teoricamente, as considerações são referentes aos textos selecionados e não podem ser generalizadas. 
PALAVRAS-ChAVE: Talento. Gestão de pessoas. Tema emergente.

\title{
Analysis of Talent in Different Areas of Knowledge
}

\begin{abstract}
The current market consists a complex, highly competitive and fragile global environment to organizations. This reality brings challenges and also, global flows of talents and scarcity of essential competences. Thus the emergence of talent management as an international and national concern on people management. Taking in consideration the aspect of multiplicity and fluidity of the market and the dimness of talent definition in Administration theory, the interest in verify the multidisciplinary characteristic of the term emerged. So, the research problem was: What are the characteristics and propositions made by different knowledge fields regarding the term Talent? Therefore this research aimed to identify characteristics and propositions that other knowledge fields adopt regarding the term talent. This study comprehends a systematic review, realized through bibliometric and content analysis. The academic papers analyzed were selected from the web of science database with the search condition: the use of the term Talent on the title. The reading of the resulted selected texts made possible the conclusion that, according to this sample, the adjectives of most frequent use when referring and describing talent are those related to differentiation. From this point, it was possible to suggest ten propositions of future research. This study was developed theoretically, the considerations are limited to the texts selected and cannot be generalized.
\end{abstract}

KEY-WORDS: Talent. Human resource management. Emerging theme. 


\section{INTRODUÇÃo}

As economias globais influenciaram a formação de organizações internacionalizadas, integradas a uma cadeia produtiva globalizada e a amplas redes de trabalho (Eurofound, 2009). O mercado atual reflete um ambiente global, complexo, altamente competitivo e volátil para as organizações, trazendo consigo desafios, também, globais de fluxo de talentos, escassez de competências essenciais e gestão de várias gerações de empregados que trabalham simultaneamente na mesma organização (Tarique \& Schuler, 2010).

A Gestão de Talentos (GT) tem ganhado força e representatividade a partir da pesquisa da Mckinsey, "The war for talent", publicada em 1998, e vem sendo adotada no meio corporativo e na literatura profissional, mostrando-se até meados dos anos 2000 como uma configuração de práticas de definição imprecisa. As empresas consideram que essa configuração é essencial na Gestão de Recursos Humanos (GRH) contemporânea e estratégica, entretanto ainda apresenta uma aparente inconsistência teórica. Análises da literatura demonstram que também na teoria esses conceitos são pouco definidos e, por vezes, imprecisos, resultando na adoção de diferentes abordagens ou orientações de GT pelas organizações (Tarique \& Schuler, 2010). Isso faz da Gestão de Talentos um tema emergente e uma preocupação internacional e nacional de Gestão de Pessoas, ocupando as primeiras posições nas pesquisas que visam detectar tendências em gestão de pessoas como a SHRM Workplace Forecast - The Top Workplace Trends (SHRM, 2011) e Delphi RH 2010 (Fischer \& Albuquerque, 2010). Nesse sentido, diversos estudos foram realizados nos anos seguintes, por meio dos quais já se pode afirmar que a GT vem se consolidando ao longo do século XXI como uma configuração de práticas para a gestão dos Talentos nas organizações (Freitag \& Fischer, 2013b).

As organizações contemporâneas são desafiadas constantemente pelas tendências em Gestão de Pessoas que estão associadas ao fenômeno da globalização e à consequente intensificação da complexidade entre as firmas (Eurofound, 2009). Considerando-se esse aspecto de multiplicidade e fluidez do mercado atual e de imprecisão do termo Talento em Administração, surgiu o interesse de verificar, também, a multidisciplinaridade desse termo. Isto é, 
verificar o Talento em diferentes áreas do conhecimento para captar as perspectivas teóricas a respeito do termo. Então, emergiu o problema de pesquisa que norteou este estudo: Quais as características e as proposições de diferentes áreas do conhecimento acerca do termo Talento?

A fim de colaborar com o debate sobre temas emergentes em Gestão de Pessoas, o objetivo desta pesquisa consiste em identificar características e proposições que outras áreas do conhecimento adotam para referir-se a Talento. Acredita-se que as diferentes perspectivas encontradas possam vir a auxiliar uma melhor compreensão e desenvolvimento do termo Talento nas pesquisas de Gestão de Pessoas e nas práticas organizacionais, resultando na sugestão de proposições para teste empírico em futuras pesquisas em Administração.

Na próxima seção, como referencial teórico deste estudo, consta uma explanação sobre Talento e Gestão de Talentos no campo da Administração, seguida das seções de Aspectos Metodológicos, Apresentação e Análise dos Resultados, Considerações Finais e Referências.

\section{TALENTO E GESTÃO DE TALENTOS NO CAMPO DA ADMINISTRAÇÃO}

Talento, para McClelland, Baldwin, Bronfenbrenner e Strodtbeck (1958), é um termo ambíguo: às vezes se refere a uma atitude ou capacidade da pessoa e outras vezes à sua performance talentosa, isto é, a um comportamento que vai além do comum. No campo da gestão, entende-se que a definição desse termo é fluida e influenciada pela estratégia organizacional e pelas contingências do ambiente em que dada organização se encontra (Ingham, 2006; Mucha, 2004).

Freitag e Fischer (2013b) analisaram definições de talento e gestão de talentos na produção acadêmica nacional e internacional de Administração de 2001 a 2011, bem como a sua utilização em uma amostra de organizações que atuam nacionalmente. Como resultado, captaram uma série de definições conceituais de talento no campo de estudo da gestão, tais como:

a) "talento é a combinação do conjunto de habilidades, competências e experiências necessárias para o desempenho no trabalho" (Berger \& 
Berger, 2004, p. 233, tradução livre);

b) "talento é igual a competência vezes comprometimento vezes contribuição" (Ulrich, 2007, p. 32, tradução livre); ou

c) "talento é a soma das habilidades do indivíduo - suas características inatas, habilidades, conhecimento, experiência, inteligência, julgamento, atitude e personalidade. Incluindo a habilidade para aprender e crescer" (Handfield-Jones \& Axelrod, 2001, p. xii, tradução livre).

Contudo, também para aqueles pesquisadores não foi possível encontrar uma definição única para Talento ou que fosse adotada frequentemente por pesquisadores de diversas áreas de pesquisa. A interpretação dos termos identificados nos textos examinados por Freitag e Fischer (2013b) permitiu consolidá-los nos atributos qualificadores de talento no campo da Administração, que seriam: competência, capacidade, habilidade, conhecimento, resultado, desenvolvimento, potencial e valores.

Berger e Berger (2004) afirmam que uma gestão proativa de talentos, que proporcione uma performance competitiva da empresa no mercado, somente pode ser obtida a partir da implantação de um Sistema de Gestão de Talentos, composto pelas seguintes práticas: identificação, seleção, desenvolvimento e retenção de talentos (Berger \& Berger, 2004; Sturman, Trevor, Boureau \& Gerhart, 2003). A identificação dos talentos é a prática pela qual se inicia o Sistema de Gestão de Talentos, pois é a partir da definição de Talento para a organização que se pode compor uma linguagem comum para nortear esse sistema (Ferreira, 2008; Ingham, 2006; Tanure, Evans \& Pucik, 2007).

No aspecto de identificação do talento, McClelland et al. (1958) propuseram uma análise baseada em quatro pilares de determinantes que levariam a uma performance considerada talentosa. Os pilares são:

a) condições antecedentes: hereditariedade, valores culturais, estrutura familiar, condição socioeconômica, perfil dos pais;

b) características pessoais: habilidades, estabilidade emocional, valores, motivação, traços ou padrões de resposta;

c) contexto: condições de trabalho, habilidades requeridas, valores requeridos, objetivos e estabilidade; 
d) resultados esperados: eficiência no trabalho, posição ocupacional, notas na escola, salário, serviços comunitários, ética, sucesso em liderança e efetividade social.

A GT vem se consolidando ao longo do século XXI como uma configuração de práticas para a gestão dos Talentos nas organizações (Freitag \& Fischer, 2013b). Nos estudos na área de Gestão de Pessoas, pode-se verificar que a GT se manifesta, basicamente, nas modalidades inclusiva ou exclusiva. Na modalidade inclusiva de GT, todos os profissionais são considerados como detentores de algum talento e, na exclusiva, trabalha-se com a diferenciação e a segmentação das pessoas de acordo com seu potencial, performance ou sua posição hierárquica na organização (Freitag \& Fischer, 2013a; Iles, Chuai \& Preece, 2010; Lewis \& Heckman, 2006).

Essa foi uma explanação sobre as principais características acerca do termo Talento encontradas na literatura acadêmica no campo da Administração, em especial, na área de Gestão de Pessoas. Na próxima seção, constam os procedimentos metodológicos utilizados para selecionar textos de diferentes áreas do conhecimento que tratem de Talento.

\section{PROCEDIMENTOS METODOLÓGICOS}

Os procedimentos metodológicos realizados neste estudo estão estruturados da seguinte forma: delineamento metodológico, procedimento de seleção da fonte de pesquisa, definição do procedimento de coleta, seleção e análise dos artigos.

\subsection{DELINEAMENTO METODOLÓGICO}

Este estudo caracteriza-se como uma revisão sistemática que objetiva uma consolidação conceitual de um campo fragmentado e que teoricamente deve ser realizada a partir da coleta sistemática de dados, análise descritiva e qualitativa, culminando com a elaboração de uma síntese fundamentada 
(Crossan \& Apaydin, 2010; Tranfield, Denyer \& Smart, 2003). Neste caso, optou-se por análise bibliométrica e análise de conteúdo.

Segundo Macias-Chapula (1998), a pesquisa bibliométrica pode ser caracterizada quanto ao objeto de estudo e ao método empregado: o primeiro, relativo à base de dados, livros, documentos, artigos, autores, etc.; o segundo, à frequência e à distribuição. Alvarenga (1998) cita que os resultados encontrados nesse tipo de pesquisa refletem aspectos quantitativos de áreas do conhecimento, apresentando, por exemplo, ângulos de produtividade de autores ou de fontes e constatações de regularidades que podem resultar em fatos históricos no processo de evolução de uma disciplina.

Na análise de conteúdo dos artigos, consta o caráter qualitativo desse estudo. A análise de conteúdo baseia-se na inferência do pesquisador sobre o que é comunicado por meio de diferentes formas de comunicação (Bardin, 1977). De acordo com Triviños (1987), essa técnica consiste em um conjunto de procedimentos sistemáticos que objetivam descrever o conteúdo de mensagens e obter indicadores quantitativos ou não, permitindo a inferência de conhecimentos sobre a condição da produção. Para tanto, esse autor propõe três fases: a pré-análise, a descrição analítica e a interpretação referencial. Neste caso, a pré-análise foi realizada com os textos encontrados; a descrição analítica permitiu a categorização por área do conhecimento; e a interpretação foi direcionada para os textos que traziam uma definição acerca do que é Talento.

Nesta revisão, realizou-se um levantamento dos artigos publicados na base Web of Science, optou-se por analisar a frequência e a distribuição dos textos encontrados por área do conhecimento, ano e quantidade de citações, além da análise de conteúdo dos textos selecionados. No que se refere ao horizonte de tempo desta pesquisa, foi utilizada a opção disponível na base Web of Science para captar todos os artigos publicados desde 1988.

\subsection{PROCEDIMENTO DE SELEÇÃO DA FONTE DE PESQUISA}

Neste estudo, optou-se por pesquisar a publicação de artigos e analisar a sua frequência e distribuição por área, ano e quantidade de citações. A fonte para esta revisão foi selecionada de modo a trazer para este estudo a produção 
de melhor classificação, aceitação no meio acadêmico e que fornecesse a possibilidade de agregar a produção de diferentes áreas do conhecimento. Isso resultou na seleção da base Web of Science como fonte desta revisão.

\subsection{PROCEDIMENTO DE COLETA, SELEÇÃO E ANÁLISE DOS ARTIGOS}

O levantamento da produção acadêmica sobre Talento em diferentes áreas do conhecimento fundamentou-se no seguinte processo de coleta $\mathrm{e}$ seleção de artigos:

a) busca na base Web of Science usando os termos de busca: talento e talent;

b) seleção por título que contivesse pelo menos um dos termos de busca, o que resultou em 3.634 registros de trabalhos pré-selecionados no período de janeiro de 1998 a dezembro de 2011;

c) aplicação de filtros para refinar a seleção da seguinte forma:

i. seleção de trabalhos classificados como artigos acadêmicos. Resultou em 1.443 artigos selecionados;

ii. lista das principais áreas temáticas de acordo com a publicação sobre Talentos. Optou-se por selecionar as dez áreas temáticas de maior publicação sobre o tema, resultando em 955 artigos;

iii. seleção, dentre os 955 artigos, aqueles que tivessem mais de 50 citações registradas na base Web of Science. Foram selecionados 20 artigos. Destes, descartaram-se cinco, pois quatro referiam-se a componentes médicos e não a indivíduos e o quinto não estava disponível para download na base pesquisada;

iv. seleção, dentre os 15 textos, os que tivessem citações acima da média. Resultou em oito artigos.

d) categorização dos trabalhos selecionados por tema no software de organização de referências, Mendeley.

O volume de artigos encontrados foi analisado bibliometricamente de acordo com Macias-Chapula (1998) e Alvarenga (1998) a partir da frequência e distribuição dos seguintes critérios: área, ano e quantidade de citações (Tabela 2). 
A fase de análise de conteúdo da produção acadêmica sobre Gestão de Talentos foi realizada com o objetivo de captar características e proposições de Talento que vêm sendo adotadas em diferentes campos de estudo. Para tanto, foram realizadas as três fases propostas por Triviños (1987): a préanálise, a descrição analítica e a interpretação referencial. Neste caso, a préanálise foi realizada com os textos selecionados por meio da leitura dos resumos. A descrição analítica resultou no agrupamento dos oito artigos de acordo com a existência ou menção do termo Talento com adjetivos, definição de Talento ou uso do termo Talento somente no título do artigo (Tabela 1 ).

A interpretação referencial foi realizada a partir do último filtro de refinamento da seleção dos textos (Tabela 2). Os 20 textos restantes para a análise foram lidos em profundidade e categorizados de acordo com a área do conhecimento em que foram publicados. Com isso, pretende-se apresentar um panorama da produção acadêmica sobre Talento em diferentes áreas do conhecimento. Na seção seguinte, constam os resultados e as análises resultantes desta pesquisa.

\section{APRESENTAÇÃO E ANÁLISE DOS RESULTADOS}

Inicialmente, procurou-se pelo termo "Talent" no título dos trabalhos publicados na base Web of Science, onde se encontraram 3.634 registros de trabalhos cujos títulos continham essa palavra específica. Os artigos apresentados são de janeiro de 1998 a dezembro de 2011. Entretanto, foi nos anos 2000 que se começa a observar um aumento quantitativo mais expressivo dessas publicações e citações, com extremos a partir de meados dessa década e uma tendência crescente de 2010 em diante.

Com o propósito de refinar a pesquisa, foi utilizado um filtro para selecionar somente os artigos acadêmicos na base selecionada. Isso resultou em 1.443 registros de artigos sobre Talento. Em seguida, listaram-se as principais áreas temáticas de acordo com a publicação sobre esse tema. Optou-se então por selecionar as dez áreas temáticas de maior publicação sobre Talentos.

As principais áreas de publicação sobre Talentos são, respectivamente, 
de educação (246), psicologia (232), gestão (Management e Business) (123), música (67), economia (66), literatura (58), esporte (54), arte (53), área médica (40), multidisciplinar (32).

Analisando a produção de cada área em separado, obtém-se a soma de 971 artigos. Entretanto, ao considerá-la de forma agregada, já que alguns trabalhos constam registrados em mais de uma área simultaneamente, a produção total dessa seleção das dez principais áreas consiste em 955 artigos, conforme consta na Web of Science (2012). Como a base pesquisada traz dados sobre a publicação acadêmica internacional, nesta amostra analisada não constam trabalhos nacionais.

Após selecionar as áreas temáticas de maior produção sobre Talento, optou-se por selecionar dentre esses artigos, os que tivessem mais de 50 citações registradas na base Web of Science. Optou-se por aqueles que tivessem um grande número de citações com o objetivo de obter artigos em que o construto Talento estivesse de alguma forma sendo disseminado. Isso resultou na seleção de 20 artigos, dos quais foi necessário descartar cinco, pois quatro apresentaram o termo Talent com o sentido de um componente médico, não como uma característica do indivíduo, e um não estava disponível para download na base pesquisada. Os 15 textos restantes foram analisados nesta seção. Para tanto, realizou-se a leitura destes em busca de informações que possibilitassem desenvolver esta seção, categorizando-os por ano, fonte, tipo, enfoque, área, assunto e uso do termo de busca. A amplitude de publicação nesta amostra consiste em 48 anos (1954-2002), evidenciando uma composição de textos seminais e contemporâneos para este estudo.

Durante a pesquisa, não foi possível encontrar uma produção maior ou discrepante em algum periódico ou com algum autor, o que permite considerar que, nessa amostra, não foi possível identificar um periódico ou pesquisador que publique de forma contumaz sobre a questão dos Talentos. A maioria dos trabalhos selecionados é do tipo teórico-empírico (13) com abordagem quantitativa (11/13), um artigo era teórico e dois eram de revisões.

Quanto à área e ao assunto dos textos mais citados, verificou-se uma maior concentração de trabalhos nas áreas de esporte (5) e economia (4), seguidos pela psicologia (3), gestão (2) e educação (1). Nessa seleção, os assuntos mais frequentes foram: a identificação, a alocação, o 
desenvolvimento e a atração de talentos.

Destes 15 trabalhos analisados, quatro possuem um capítulo teórico intitulado com o termo da pesquisa (Talent/Talento). Na Tabela 1, pode-se observar que, na maioria desses textos, somente foi mencionada a palavra Talento ao longo do trabalho sem, necessariamente, defini-la conceitualmente, apresentando-a acompanhada somente de adjetivos e outros termos cognatos para auxiliar na compreensão do assunto. Foi possível encontrar somente uma definição conceitual para talento dentre os artigos analisados.

Tabela 1: Artigos mais citados sobre Talento, de acordo com o uso do termo de busca

\begin{tabular}{l|r}
\multicolumn{1}{c|}{ Classificação } & Freq. \\
\hline Menção a Talento com adjetivos & 11 \\
\hline Definição de Talento & 2 \\
\hline Uso do termo Talento somente no título & 1 \\
\hline Total & $\mathbf{1 5}$ \\
\hline
\end{tabular}

Fonte: Análise da produção acadêmica e Web of Science (2012)

Dessa forma, pode-se inferir que não houve nesse período um esforço continuado ou intenção acadêmica de desenvolver conceitualmente o que seja Talento. Os pesquisadores analisados seguiram diretamente para assuntos relacionados às características que permitem trabalhar com esse tipo de profissional, por exemplo, identificar e desenvolver esses talentos, embora seja evidente a necessidade de maior entendimento acerca desse termo, já que há uma produção considerável sobre processos inerentes à gestão do Talento.

A fim de buscar definições conceituais ou formas de uso do termo Talento em diversas áreas do conhecimento, optou-se por refinar a seleção de textos que foi analisada até esta fase. Para tanto, aplicou-se o critério de maior citação. Por fim, os oito artigos selecionados foram organizados de acordo com ano e área temática (Tabela 2). 
Tabela 2: Textos mais citados sobre Talento em diversas áreas do conhecimento

\begin{tabular}{c|c|c|c}
\hline Área & \multicolumn{1}{c|}{ Referência } & Ano & $\begin{array}{c}\text { Times } \\
\text { Cited }\end{array}$ \\
\hline Psicologia & $\begin{array}{l}\text { Terman, L. M. (1954). The discovery and encouragement of } \\
\text { exceptional talent. The American Psychologist, 9(6), 221- } \\
\text { 230. }\end{array}$ & 1954 & 76 \\
\hline Psicologia & $\begin{array}{l}\text { Mackinnon, D. W. (1962). The nature and nurture of creative } \\
\text { talent. American Psychologist, 17(7), 484-495. }\end{array}$ & 1962 & 400 \\
\hline Economia & $\begin{array}{l}\text { Murphy, K. M., Shleifer, A., \& Vishny, R. W. (1991). The } \\
\text { allocation of talent: implications for growth. The Quarterly } \\
\text { Journal of Economics, 106(2), 503-530. }\end{array}$ & 1991 & 312 \\
\hline Educação & $\begin{array}{l}\text { Hanson, S. L. (1994). Lost talent: unrealized educational } \\
\text { aspirations and expectations among U.S. youths. Sociology } \\
\text { of Education, 67(3), 159-183. }\end{array}$ & 1994 \\
\hline Psicologia & $\begin{array}{l}\text { Howe, M. J. A., Davidson, J. W., \& Sloboda, J. A. (1998). } \\
\text { Innate talents: reality or myth? The Behavioral and Brain } \\
\text { Sciences, 21(3), 399-407. }\end{array}$ & 1998 & 147 \\
\hline Esporte & $\begin{array}{l}\text { Côté, J. (1999). The influence of the family in the development } \\
\text { of talent in sport. The Sport Psychologist, 13(4), 395-417. }\end{array}$ & 1999 & 166 \\
\hline Esporte & $\begin{array}{l}\text { Reilly, T., Williams, A. M., Nevill, A., \& Franks, A. (2000). A } \\
\text { multidisciplinary approach to talent identification in soccer. } \\
\text { Journal of Sports Sciences, 18(9), 695-702. }\end{array}$ & 2000 \\
\hline $\begin{array}{l}\text { Williams, A. M., \& Reilly, T. (2000). Talent identification and } \\
\text { development in soccer. Journal of Sports Sciences, 18(9), } \\
\text { 657-667. }\end{array}$ & 2000 & 130 \\
\hline
\end{tabular}

Fonte: Web of Science (2012)

Os textos apresentados na Tabela 2 foram analisados com a finalidade de buscar termos, características e definições sobre Talento. Além disso, as concepções sobre esse tema encontradas nesses textos foram organizadas e apresentadas na seção 4.1 no formato de proposições.

\subsection{CARACTERÍSTICAS E PROPOSIÇÕES SOBRE TALENTO EM DIFERENTES ÁREAS DO CONHECIMENTO}

A leitura dos textos selecionados possibilitou a constatação de que, de acordo com essa amostra (Tabela 2), os adjetivos mais utilizados para fazer menção e caracterizar os Talentos são os relacionados à diferenciação. Tais como: best (melhor), super, excepcional, high (alto). Os vocábulos apontados junto a estes foram: potencial, performance, habilidade, jovem, inato e gifted ("dado" - termo relacionado à característica inata de talento). No texto de 
economia, houve uma relação do talento com cargo ou posição hierárquica.

Durante a análise dos textos selecionados na Tabela 2, foi encontrada somente uma definição explícita para Talento no texto que consta no campo da Psicologia. Essa definição é original do texto de Howe, Davidson e Sloboda (1998), mas também foi citada por Williams e Reilly (2000). Segundo estes autores pode-se afirmar que:

Certas armadilhas devem ser evitadas na resolução sobre a definição de talento. Uma definição muito restrita pode tornar impossível demonstrá-lo para qualquer evidência concebível. Por exemplo, algumas pessoas acreditam que talento seja uma capacidade inata que torna certo que seu possuidor irá sobressair-se. Esse critério é muito forte. No outro extremo, seria possível fazer a definição de talento tão vaga que a sua existência seria trivialmente assegurada; talento poderia implicar que aqueles que atingem altos níveis de realização seriam de alguma forma indefinida biologicamente diferentes dos demais. (Howe et al., 1998, p. 399-400; tradução e grifo nossos).

Howe et al. (1998) consideram que a conceituação de Talento não deve ser tão restritiva, ao ponto de ser impossível encontrar uma pessoa com estas características no mercado, mas também não deve ser ampla demais para não se tornar algo trivial tal como uma característica que qualquer pessoa possa ter. Para tanto, propuseram cinco propriedades do Talento, que envolvem aspectos relacionados (1) ao potencial, (2) à precocidade, (3) à característica de ser inato, e (4) ser próprio de uma minoria com performance e resultados diferenciados, além de (5) ser relativo a um domínio específico (Howe et al., 1998).

Tais considerações possibilitaram a elaboração de proposições acerca do termo Talento, as quais foram apresentadas e discutidas de maneira que pudessem contribuir para uma reflexão sobre este tema e para uma futura agenda de pesquisa. Seguem as proposições $(P)$ :

P1: O talento está relacionado à diferenciação;

P2: O potencial de talento é captado precocemente nos indivíduos.

Howe et al. (1998) afirmam que, tal como outras nomenclaturas científicas, Talento não é um conceito observado diretamente, mas inferido. É, portanto, impreciso por natureza.

Em relação a diversos campos de estudo, Terman (1954) pesquisou aspectos relacionados à identificação e à formação de Talentos, constatando que o intelecto, o quociente de inteligência (QI) e as conquistas dos indivíduos 
estão longe de ser perfeitamente correlacionados. Entretanto, admite que padrões de interesse e aptidões especiais desempenhem papéis importantes na formação do cientista, do matemático ou do artista de Talento. Argumenta também que os talentos especiais precisam ser apoiados por uma grande capacidade do indivíduo de exercer pensamento abstrato, um tipo de inteligência geral que requer a capacidade de formar conceitos nitidamente definidos para manipulá-los percebendo relações sutis entre eles.

Terman (1954) afirma que identificar fatores externos e internos que interferem no desenvolvimento e ação dos Talentos, bem como medir a extensão dessa influência são questões que figuram entre os principais problemas que permeiam este tema. Esse pesquisador ressalta que a contemporaneidade, à época, dessa questão consiste na consciência geral da escassez de mão de obra especializada, tal como de cientistas, líderes e engenheiros.

P3: A subjetividade na identificação influencia a percepção de escassez de talentos.

No que se refere ao campo das ciências, Mackinnon (1962), ao pesquisar sobre o Talento criativo, considera que a criatividade é um processo caracterizado pela originalidade, podendo ser breve, como uma improvisação musical, ou levar décadas para ser concluído, como a formulação de uma teoria. O produto dessa criatividade está relacionado ao criador como indivíduo que, em seu trabalho criativo, atua como um mediador entre necessidades e metas definidas externamente. Portanto, nesse tipo de criatividade, o criador, ou seja, o engenheiro, o professor, o administrador, age no ambiente produzindo um produto novo ao qual adiciona um pouco de si ou do seu estilo, sendo o resultado relacionado a si. Nessa perspectiva, esse autor observou que pessoas altamente criativas tendem a ter boa opinião de si mesmas, utilizando um elevado número de adjetivos favoráveis em sua autodescrição.

Mackinnon (1962), em suas observações sobre a relação entre inteligência e criatividade, sugere que nas escolas norte-americanas pode-se ter superestimado a contribuição da inteligência para a realização criativa. Em suas pesquisas, ele não encontrou razão para acreditar que o aluno com QI mais alto seja o mais promissor e deva, portanto, ser escolhido para uma educação superior. Os resultados de sua pesquisa sugerem que, se uma 
pessoa tem o mínimo de inteligência necessária para o domínio de um campo de conhecimento, a sua ação, seja esta criativa ou banal, será fundamentalmente determinada por fatores não intelectuais e que se deve atentar para o futuro (potencial) mais do que para o passado (conquistas passadas) a fim de desenvolver essas características.

A análise dos parágrafos anteriores fez emergir as seguintes proposições para serem testadas empiricamente:

P4: O talento está relacionado à capacidade do indivíduo de exercer pensamento abstrato.

P5: Os fatores não intelectuais influenciam o potencial de talento de um indivíduo.

No campo da economia, Murphy, Shleifer e Vishny (1991) trouxeram a questão da alocação de Talento, nesse caso, do capital humano, como influenciadora no crescimento econômico de um país. Para tanto, fizeram um estudo nos Estados Unidos a fim de compreender a desaceleração do crescimento da produtividade daquele país e constataram que dois motivos deste cenário seriam relativos ao capital humano. O primeiro relaciona-se à qualidade desse capital, em que se considerou a queda no padrão da formação escolar, o declínio do uso dos testes de inteligência e a queda no salário dos profissionais com ensino médio. A segunda razão foi a alocação inapropriada desse capital humano, que pode impedi-lo de gerar crescimento econômico. Principalmente, os jovens deixaram de ser empreendedores ou engenheiros, isto é, "construtores" e inovadores, e passaram a ser rent-seekers ou "mantenedores", como advogados por exemplo. A partir de uma série de cálculos e de um modelo teórico, esses autores propuseram que, no que se refere à expectativa de crescimento econômico, as profissões "mantenedoras" seriam desaconselháveis frente às "construtoras".

Os autores pesquisados sugerem que os incentivos privados que regem a distribuição de talentos em todas as profissões podem não coincidir com incentivos sociais. Assim, algumas profissões são socialmente mais úteis do que outras, mesmo que não sejam tão bem remuneradas. Eles afirmam não saber qual é a causa exógena dessas relações. No entanto, acreditam ser possível que as políticas promotoras de investimento ou que visem melhorar a qualidade do capital humano, indiretamente, devam tornar as carreiras 
"construtoras" mais atraentes e, dessa forma, possibilitar o crescimento econômico do país (Murphy et al., 1991).

Na área temática da educação, Hanson (1994) aborda a questão da identificação precoce do talento. Para tanto, pesquisou sobre a questão dos "talentos perdidos" no âmbito dos jovens estudantes dos Estados Unidos. O autor considera que o fenômeno dos "talentos perdidos" ocorre quando estudantes que demonstram sinais precoces de talento (1) têm expectativas educacionais que ficam aquém de suas aspirações, (2) têm expectativas educacionais reduzidas ao longo do tempo, (3) não são capazes de perceber precocemente as suas expectativas. Dessas três características, Hanson (1994) constatou que a classe social exerce o maior efeito na perda desses jovens potenciais talentos. Isto é, quando o jovem é membro de uma classe de baixo poder socioeconômico, o risco da perda ou não concretização desse potencial talento na sociedade é dobrado. A partir desse texto, surgiu a seguinte proposição a ser testada empiricamente:

P6: A educação influencia no aproveitamento do potencial de talento.

Nos esportes, Côté (1999) pesquisou a influência da família no desenvolvimento dos jovens talentos. Seus estudos foram realizados com o propósito de descrever padrões que envolvem a dinâmica das famílias de atletas talentosos a partir do seu desenvolvimento. Para tanto, foram identificadas três fases da participação da família na formação desse jovem talento no esporte desde a primeira infância até a adolescência: idade 6-13, anos de experimentação (sampling years); idade 13-15, anos de especialização (specializing years); acima de 15 anos de idade, anos de investimento (investment years).

No decorrer do texto, Côté (1999) trouxe contribuições teóricas de diversos pesquisadores. Ele utilizou como base de sua pesquisa o modelo teórico de Ericsson, Krampe e Tesch-Römer (1993), no qual é sugerido que uma performance especializada envolve operar com três tipos de atributos: motivação, esforço e recursos. Uma série de pesquisadores citados sugere que o desempenho de elite no esporte é resultado de muita prática e não somente de habilidades naturais (Ericsson et al., 1993; Hodge \& Deakin, 1998). As pesquisas comentadas suscitaram a emersão das seguintes proposições:

P7: O indivíduo é capaz de desenvolver seu potencial de talento. 
P8: A motivação é potencializadora de talento.

Howe et al. (1998) defendem que as crenças dos pais sobre os talentos de seus filhos são fortes determinantes de seus comportamentos e levam a um reforço positivo no esforço de seus filhos. Ericsson et al. (1993) foram citados para reforçar que o ambiente familiar é uma variável importante que afeta o desenvolvimento do talento em muitos domínios, incluindo o dos esportes.

Os resultados da pesquisa de Côté (1999) apontaram que a principal razão para um adolescente escolher especializar-se em uma atividade reside no valor positivo associado àquela experiência. Alguns pais entrevistados acreditam que, para alcançar uma alta performance, seja no esporte ou na música, o jovem teria que ter primeiramente algum talento natural. Diante desses resultados e dos autores citados em seu texto, esse autor constatou que, para reforçar a compreensão da influência da família no desenvolvimento de uma elite no esporte, o ambiente familiar precisa ser estudado por completo em cada fase do desenvolvimento da criança combinado a outras perspectivas relacionadas à formação de talentos. Por fim, Côté (1999) afirma que o papel da família no envolvimento das crianças no esporte é um fenômeno complexo devido à diversidade do contexto familiar. Diante disso, segue a próxima proposição para ser testada empiricamente:

P9: A crença da família no talento do indivíduo reforça positivamente o seu desenvolvimento.

Williams e Reilly (2000) trazem uma revisão da literatura com o propósito de integrar pesquisas sobre identificação e desenvolvimento de talento no futebol. Consideraram abordagens antropométricas, fisiológicas, psicológicas e sociológicas. Alguns progressos já foram realizados na identificação de jogadores de sucesso, mas não parece ter características únicas que possam ser isoladas. Poucas são as pessoas de talento em um domínio específico, se todas as crianças tivessem o mesmo talento não seria possível explicar o diferencial de sucesso na vida e carreira de cada uma. Logo, esses autores afirmam que Talento é específico a um determinado domínio. Essas características auxiliam a compreender a complexidade da natureza do talento e retratam porque não há consenso a respeito da teoria e prática na identificação de talento nos esportes. Com isso, sugere-se a seguinte 
proposição para ser testada empiricamente:

P10: O talento é particular a um contexto específico.

Williams e Reilly (2000) sugerem em sua pesquisa alguns preditores de talento para o esporte e que não estariam concentrados essencialmente em características intelectuais, mas, sim, em uma abordagem multidisciplinar, contemplando atributos físicos, fisiológicos, sociológicos e psicológicos. Nesse contexto, eles citam que os jogadores de melhor qualificação, quando comparados aos demais, são: mais rápidos e ágeis em reconhecer padrões de jogo, melhores em antecipar as jogadas do oponente e mais precisos quanto às expectativas de acontecimentos prováveis frente a determinado conjunto de circunstâncias em campo. A partir dessas características, é possível observar duas medidas cognitivas que vêm sendo propostas como preditores de talento: inteligência e pensamento criativo. Entretanto, não está claro se a inteligência de jogo está relacionada à acadêmica. Também, não está definido se as conclusões acerca dos jogadores do gênero masculino podem ser estendidas para o gênero feminino. A complexidade do talento e as questões metodológicas associadas à sua identificação excluem a utilização de uma abordagem monodisciplinar. Portanto, os autores concluem que uma tendência promissora, nesse campo de estudo, são as abordagens multidisciplinares, que seriam mais estruturadas e holísticas (Williams \& Reilly, 2000).

No que se refere a uma abordagem multidisciplinar para identificação de talentos nos esportes cabe inserir a contribuição do texto de Reilly, Williams, Nevill e Franks (2000), que aplicaram uma série de testes em jovens jogadores de futebol a fim de encontrar distinções de performance entre os grupos de elite e de subelite. Técnicas de análise de estatística multivariada foram utilizadas para determinar que combinação de medidas mais claramente diferenciaria os dois grupos de jogadores. As análises revelaram que os grupos podem ser discriminados com base em quatro medições: agilidade, velocidade, orientação motivacional e habilidade de antecipação. Estas parecem ser os mais fortes preditores de talento. Os autores ressaltam no texto que a capacidade de "ler o jogo" e antecipar as intenções do adversário é uma característica importante dos jogadores talentosos. Reilly et al. (2000) concluem seu estudo propondo uma fase longitudinal da pesquisa a fim de que seja possível especificar se as medidas propostas serviriam mais para 
diferenciar os talentos do que para identificá-los.

Williams e Reilly (2000) consideram que o processo de encontrar jogadores de excelência estaria dividido em quatro fases: detectar, selecionar, identificar e desenvolver. A diferença entre detectar e identificar é que a primeira fase consiste em descobrir uma pessoa de potencial para o esporte e a segunda refere-se ao reconhecimento nos atuais participantes de um com potencial para tornar-se jogador de elite. Eles citam que contribuições acadêmicas e científicas podem auxiliar nesse julgamento, trazendo um grau de objetividade para esse processo. Os autores encerram esse texto afirmando que, no processo de identificação e desenvolvimento de talentos nos esportes, deve-se zelar pelo desenvolvimento global da criança e pelo seu bem-estar. É primordial a consciência de que a busca pela excelência não deve ocorrer à custa da saúde física e emocional desses potenciais talentos. Uma abordagem fundamentada em um ambiente familiar, educacional e sociocultural apropriado e equilibrado é a base para o desenvolvimento de uma criança, especialmente quando se trata da formação de jogadores de elite.

Essas foram as características e proposições acerca de Talento encontradas nos textos mais citados sobre este tema disponíveis na base Web of Science em diferentes áreas do conhecimento. Na próxima seção, constam as considerações finais deste estudo.

\section{CONSIDERAÇÕES FINAIS}

O termo Talento é tratado por pesquisadores da área de Administração como impreciso e ambíguo, sendo influenciado pelas contingências do ambiente e pelas estratégias organizacionais. Embora, essa condição venha se difundindo entre os acadêmicos da área, o questionamento sobre o que venha a ser talento ainda é presente e guiou este estudo.

Ao abordar o tema Talento, que figura entre as principais preocupações dos gestores da área de acordo com estudos nacionais e internacionais já citados, este estudo foi conduzido com a intenção de colaborar com o debate sobre as tendências de Gestão de Pessoas. Dessa forma, o objetivo foi identificar características e proposições que outras áreas do conhecimento 
adotam para referir-se a Talento. Para isso, foi realizado um levantamento da produção acadêmica sobre Talento na base Web of Science, por meio do qual foram selecionados os textos com maior citação (Tabela 2) para que fossem analisados neste estudo.

No campo da Administração, os termos mais utilizados para caracterizar Talento são os determinantes classificados como antecedentes, perfil pessoal, contexto e resultados, tais como: hereditariedade, valores culturais, estrutura familiar, condição socioeconômica, performance, competência, habilidades, estabilidade emocional, motivação, condições de trabalho, resultado, desenvolvimento, potencial, eficiência no trabalho, posição ocupacional, notas na escola, salário, serviços comunitários, ética, sucesso em liderança e efetividade social. Nos textos de diferentes áreas do conhecimento, verificaram-se as seguintes características para referir-se a Talento: QI, sucesso, potencial, contexto social e familiar, ambiente, aspectos físicos, fisiológicos e de personalidade.

A imprecisão conceitual parece ser inerente ao termo Talento, mostrando-se evidente mesmo quando se estudam diferentes áreas do conhecimento. Portanto, ao analisar o material reunido neste estudo, acreditase que, ainda, não seja possível responder com uma única definição o termo Talento, de maneira que ele seja apropriadamente aplicado em todas as áreas do conhecimento. Essa constatação corrobora os achados teóricos deste estudo, pois, desde 1954, Terman vinha sugerindo na Psicologia que meios de mensurar e identificar os talentos seriam as principais dificuldades desse tema. Em 2000, Williams e Reilly afirmaram que uma abordagem monodisciplinar não seria suficiente, sendo necessário envolver aspectos físicos, fisiológicos, sociológicos e psicológicos para atuar na identificação dos talentos. McClelland et al. (1958) já diziam que se tratava de um termo ambíguo e impreciso.

Diante da explanação sobre Talento e Gestão de Talentos no campo da Administração e da análise dos textos sobre Talento em diferentes áreas do conhecimento, foi possível sugerir proposições para que sejam verificados futuramente os relacionamentos entre características e dimensões de talento evidenciadas neste estudo, que poderão ser testadas empiricamente em futuras pesquisas em diversas áreas do conhecimento e, em especial, no campo da Gestão de Pessoas: P1: O talento está relacionado à diferenciação; 
P2: O potencial de talento é captado precocemente nos indivíduos; P3: A subjetividade na identificação influencia na percepção de escassez de talentos; P4: O talento está relacionado à capacidade do indivíduo de exercer pensamento abstrato; P5: Os fatores não intelectuais influenciam o potencial de talento de um indivíduo; P6: A educação influencia no aproveitamento do potencial de talento; P7: O indivíduo é capaz de desenvolver seu potencial de talento; P8: A motivação é potencializadora de talento; P9: A crença da família no talento do indivíduo reforça positivamente o seu desenvolvimento; P10: O talento é particular a um contexto específico.

A discussão teórica apresentada resultou na sugestão de proposições que, quando testadas, podem vir a auxiliar nas práticas de Gestão de Pessoas que focam a identificação dos talentos nas e para as organizações, haja vista, que se trata de um construto inferido, fluido e inerente ao meio em que está inserido. Acredita-se que as proposições apresentadas possam vir a despertar o interesse em pesquisas que venham a aprofundar o tema, contribuindo para o desenvolvimento deste conhecimento no Brasil, e futuramente afetar ações dos tomadores de decisão em Gestão de Pessoas. Quanto às limitações, cabe enfatizar que este estudo foi desenvolvido teoricamente, as considerações resultantes são referentes aos textos selecionados e não podem ser generalizadas. 


\section{REFERÊNCIAS}

Alvarenga, L. (1998). Bibliometria e arqueologia do saber de Michel Foucault: traços de identidade teórico-metodológica. Revista Ciência da Informação - Ci. Inf. [online], 27(3).

Bardin, L. (1977). Análise de conteúdo. Lisboa: Edições 70.

Berger, L. A., \& Berger, D. R. (2004). The talent management handbook: creating organizational excellence by identifying, developing, and promoting your best people. New York: McGraw-Hill.

Côté, J. (1999). The influence of the family in the development of talent in sport. The Sport Psychologist, 13(4), 395-417.

Crossan, M. M., \& Apaydin, M. (2010). A multi-dimensional framework of organizational innovation: a systematic review of the literature. Journal of Management Studies, 47(6), 1154-1191.

Ericsson, K.A., Krampe, R. T., \& Tesch-Römer, C. (1993). The role of deliberate practice in the acquisition of expert performance. Psychological Review, $100(3), 363-406$.

European Foundation for the Improvement of Living and Working Conditions Eurofound. (2009). Europe at work: better life and opportunities for all. Fouryear work programme: 2009-2012.

Ferreira, A. M. (2008). Políticas e práticas de gestão de pessoas como fatores de retenção de jovens talentos: um estudo com trainees de empresa brasileira do setor siderúrgico. Dissertação de Mestrado, Pontifícia Universidade Católica de Minas Gerais, Belo Horizonte, MG, Brasil..

Fischer, A. L., \& Albuquerque, L. G. (Coord.). (2010). Relatório Delphi-RH 2010: tendências em gestão de pessoas nas empresas brasileiras. São Paulo: PROGEP-FIA.

Freitag, B. B., \& Fischer, A. F. (2013a). Programas formais de gestão de talentos no Brasil: uma realidade? Anais do Encontro da Associação Nacional de PósGraduação e Pesquisa em Administração - ENANPAD, 37, Rio de Janeiro, RJ, Brasil.

Freitag, B. B., \& Fischer, A. F. (2013b). Talentos em gestão e gestão de talentos: análise da literatura acadêmica e de práticas corporativas. Anais dos Seminários em Administração - Semead, 16, São Paulo, SP, Brasil.

Handfield-Jones, H., \& Axelrod, B. (2001). The war for talent. Boston: Harvard Business School Press.

Hanson, S. L. (1994). Lost talent: unrealized educational aspirations and expectations among U.S. youths. Sociology of Education, 67(3), 159-183. 
Hodge, T.; Deakin, J. M. (1998). Deliberate practice in the martial arts: the role of context in motor recall. Journal of Sport and Exercise Psychology, 20(3), 260-279.

Howe, M. J. A., Davidson, J. W., \& Sloboda, J. A. (1998). Innate talents: reality or myth? The Behavioral and Brain Sciences, 21(3), 399-407.

Iles, P., Chuai, X., \& Preece, D. (2010). Talent management and HRM in multinational companies in Beijing: definitions, differences and diversity. Journal of World Business, 45(2), 179-189.

Ingham, J. (2006). Closing the talent management gap. Strategic HR Review, $5(3), 20-23$.

Lewis, R. E., \& Heckman, R. J. (2006). Talent management: a critical review. Human Resource Management Review, 16(2), 139-154.

Macias-Chapula, C. A. (1998). O papel da informetria e da cienciometria e sua perspectiva nacional e internacional. Revista Ciência da Informação - Ci. Inf. [online], 27(2), 134-140.

Mackinnon, D. W. (1962). The nature and nurture of creative talent. American Psychologist, 17(7), 484-495.

McClelland, D. C., Baldwin, A. L., Bronfenbrenner, U., \& Strodtbeck, F. L. (1958). Talent and society: New perspectives in the identification of talent. New Jersey: Princeton.

Mucha, R. T. (2004). The art and science of talent management. Organizational Development Journal, 22(4), 96-100.

Murphy, K. M., Shleifer, A., \& Vishny, R. W. (1991). The allocation of talent: implications for growth. The Quarterly Journal of Economics, 106(2), 503-530.

Reilly, T., Williams, A. M., Nevill, A., \& Franks, A. (2000). A multidisciplinary approach to talent identification in soccer. Journal of Sports Sciences, 18(9), 695-702.

Society for Human Resource Management - SHRM. (2011). Workplace forecast. Recuperado em 10 de dezembro, 2012, de https://www.shrm.org/hrtoday/news/hr-magazine/documents/11-0014wpf posting 6.pdf

Sturman, M. C., Trevor, C. O, Boudreau, J. W., \& Gerhart, B. (2003). Is it worth it to win the talent war? Evaluating the utility of performance-based pay. Personnel Psychology, 56(4), 997-1035.

Tanure, B., Evans, P., \& Pucik, V. (2007). A gestão de pessoas no Brasil: virtudes e pecados capitais. Rio de Janeiro: Elsevier.

Tarique, I., \& Schuler, R. S. (2010). Global talent management: Literature review, integrative framework, and suggestions for further research. Journal of World Business, 45(2), 122-133. 
Terman, L. M. (1954). The discovery and encouragement of exceptional talent. The American Psychologist, 9(6), 221-230.

Tranfield, D., Denyer, D., \& Smart, P. (2003). Towards a methodology for developing evidence-informed management knowledge by means of systematic review. British Journal of Management, 14(3), 207-222.

Triviños, A. N. S. (1987). Introdução à pesquisa em ciências sociais: a pesquisa qualitativa em educação. São Paulo: Atlas.

Ulrich, D. (2007). The talent trifecta. Workforce Management, 86(15), 32-33.

Web of Science. (2012). Base de dados da ISI Web of knowledge. Recuperado em 17 de janeiro, 2012, de http://images.webofknowledge.com/WOK40/help/WOK/h_database.html\#ccr

Williams, A. M., \& Reilly, T. (2000). Talent identification and development in soccer. Journal of Sports Sciences, 18(9), 657-667. 